\title{
Supraventricular tachycardia in infants: response to initial treatment
}

\author{
N Sreeram, C Wren
}

\begin{abstract}
All patients with supraventricular tachycardia during the first 12 months of life who presented between 1977 and 1988 were identified by a retrospective survey of records in this hospital and by a questionnaire sent to paediatricians in the Northern region. Twenty two of 29 patients $(76 \%)$ were in heart failure and seven $(24 \%)$ had cardiogenic shock. Seven patients $(24 \%)$ were free of symptoms. All had narrow QRS tachycardia at 215-315 beats/minute (mean (SD) 292 (21)). Initial treatment included digoxin (effective in seven of 14 patients, with overdose in three), verapamil (effective in three of three but fatal in one), cardioversion (effective in all 10 who were treated in this way), iced water applied to the face (effective in all 16 patients on 53 of 59 occasions, $90 \%$ ). Initial treatment in local hospitals was less effective and associated with more complications than that given in the regional referral centre. Digoxin is often ineffective, return to sinus rhythm is delayed, and overdosing is common. Cardioversion is effective but tachycardia often recurs. Iced water is safe and effective, and should become the treatment of choice for termination of supraventricular tachycardia in neonates and young infants.
\end{abstract}

Supraventricular tachycardia in early infancy is dangerous and potentially fatal, but if it is diagnosed early and treated appropriately it has an excellent prognosis. Until a few years ago the recommended initial treatment was usually either digoxin or cardioversion. Digoxin, however, was often ineffective, and cardioversion required anaesthesia or sedation and was Many newer forms of treatment have been proposed by the authors of standard textbooks on paediatrics including verapamil, disopyramide, and propranolol given intravenously. ${ }^{1-4}$ More recently the dangers of some of these treatments have been recognised, particularly the use of verapamil in neonates. ${ }^{5} 6$ This study assesses the safety and efficacy of initial treatment of tachycardia in infancy.

Paediatric Cardiology, Freeman Hospital, Newcastle upon Tyne $\mathrm{N}$ Sreeram

C Wren

Correspondence to: Dr C Wren, Department of Paediatric Cardiology Freeman Hospital, NE7 7DN.

Accepted 4 August 1989

\section{Patients and methods}

The records of all infants presenting with supraventricular tachycardia to the Northern Regional Paediatric Cardiology Unit between October 1977 and November 1988 were reviewed. A questionnaire was sent to all paediatricians working within the Northern region to not easily repeated if the tachycardia recurred.

identify patients who were not referred to this centre. Infants with prenatal tachycardia that recurred after delivery were included. Those who were treated prenatally but who received no treatment after delivery and had no postnatal recurrence of tachycardia were excluded. Patients whose only arrhythmias were atrial flutter or multifocal atrial tachycardia were also excluded. The clinical history, physical examination, and the electrocardiograms taken during tachycardia and during sinus rhythm were available for all patients. The treatments, their efficacy, and their side effects were recorded. All survivors are still being followed up, but these details are not included as they do not form part of this study.

Application of cold water to the face elicits the 'diving reflex' and produces a temporary pronounced increase in vagal tone, thus slowing atrioventricular conduction and interrupting the tachycardia. ${ }^{7}$ The use of this technique in neonates was first described in 1977 but it is still not widely used. ${ }^{8}$ Application of an ice cold facecloth has been recommended but is often ineffective. ${ }^{6}$ In our experience the best technique is to attach the baby to an electrocardiographic monitor, wrap him in a towel, and then immerse his whole face in iced water for about five seconds. No attempt is made to obstruct the mouth or nostrils as the baby will be temporarily apnoeic. The tachycardia usually stops immediately but cessation may be delayed for two or three seconds or may occur after the baby's head has been removed from the water. It is usually followed by bradycardia, often a nodal escape rhythm, for a few seconds before resumption of normal sinus rhythm.

\section{Results}

INCIDENCE

Twenty nine patients who had supraventricular tachycardia during the first 12 months of life were identified. Twenty five of these were seen at the Freeman Hospital, and the remaining four were diagnosed and treated at local hospitals. From 1977 to 1988 the birth rate in the Northern region was relatively constant at about 40000 live births a year (range $39350-41$ 101) giving an incidence of infant supraventricular tachycardia of 1/15 000 live births.
All 29 patients presented within the first three months of life and $19(66 \%)$ were seen within the first month. Twenty two (76\%) had symptoms and clinical signs of congestive cardiac 
failure. Seven of these were in cardiogenic shock and required active resuscitation. Three had had cardiac arrests in their referring hospitals and although two were successfully resuscitated, both have been left with pronounced developmental or physical problems. The remaining seven patients $(24 \%)$ were free of symptoms and had no signs of cardiac failure.

\section{ELECTROCARDIOGRAPHY}

During tachycardia the surface 12 lead electrocardiogram tracings showed regular, narrow QRS tachycardia in every case, with heart rates varying from 215 to $315 /$ minute (mean (SD) 292 (21)). There was no correlation between heart rate and the presence of symptoms. The tachycardia rate was above 270 /minute in all but two patients; they had rates of 215 and 250 /minute and were both premature, being born at 32 and 33 weeks' gestation, respectively. Two other babies were born prematurely, at 31 and 32 weeks' gestation respectively, but both had tachycardias of $300 /$ minute. On reversion to sinus rhythm the electrocardiograms showed the ventricular prexcitation that is characteristic of the Wolff-Parkinson-White syndrome in 16 cases $(55 \%)$; they were normal in the other patients.

\section{INITIAL TREATMENT \\ Digoxin}

Fourteen infants received digoxin initially, seven intravenously and seven intramuscularly. The doses ranged from 25 to $600 \mu \mathrm{g}(8-160 \mu \mathrm{g} /$ $\mathrm{kg}$ ) and three patients received appreciable overdoses $(375-600 \mu \mathrm{g})$. Half reverted to sinus rhythm after a mean of 13 hours (range $<1$ to 36) and there was early recurrence of tachycardia in three of these. In the remainder, in whom the tachycardia persisted, sinus rhythm was restored either with cardioversion or with iced water applied to the face.

\section{Cardioversion}

Sinus rhythm was restored immediately in all 10 babies treated with electrical cardioversion but tachycardia recurred during the same hospital admission in four patients, all of whom had also received digoxin. Cardioversion was performed with 5-50 J. One patient received seven shocks (totalling $140 \mathrm{~J}$ ), which produced alarming arrhythmias varying from ventricular tachycardia to transient complete heart block during the next few hours.

\section{Verapamil}

Three patients were given verapamil. Sinus rhythm was restored in all three but one patient inadvertently received an overdose of $1.5 \mathrm{mg}$ $(0.4 \mathrm{mg} / \mathrm{kg})$ resulting in cardiac arrest and death.

Iced water applied to the face

Sixteen patients were treated by application of ice cold water to the face on 59 occasions. This was mainly by immersion of the face in ice cold water, although ice packs were applied on several occasions. The procedure was effective in restoring sinus rhythm in 53 of 59 attempts $(90 \%)$ and in all 16 patients. Tachycardia recurred at least once in 11 of the 16 patients (69\%). There were no side effects.

\section{Adenosine}

One patient was given an intravenous bolus dose of adenosine $(100 \mu \mathrm{g} / \mathrm{kg})$, and sinus rhythm was restored immediately. There were no side effects.

TREATMENT AT THE REFERRING HOSPITAL

Twenty two patients received their initial treatment in the local hospital; most were then referred to Freeman Hospital for further assessment. Fifteen were given digoxin and three (20\%) received serious overdoses. Cardioversion was carried out in three patients, one of whom received a serious overdose (see above). Two patients were given verapamil, one of whom died (see above). Nine patients were treated with iced water applied to the face, and sinus rhythm was restored in six $(66 \%)$. Tachycardia persisted in the other three and sinus rhythm was subsequently restored by further applications of iced water in Freeman Hospital. This result may be accounted for by the district paediatricians' lack of familiarity with the procedure, which can cause anxiety in an inexperienced operator.

\section{OUTCOME}

Twenty six of the 29 patients were alive and well at the time of writing. One patient died after being given verapamil. One patient is awaiting renal transplantation for polyuric renal failure secondary to the initial cardiogenic shock. A further patient developed severe cerebral anoxia and now has profound developmental delay. In this last case it is possible, in retrospect, to identify a delay in diagnosis of several hours after presentation to the district hospital.

\section{Discussion}

The diagnosis of supraventricular tachycardia in infants is not always straightforward, and its importance is not always fully appreciated. With two exceptions, the heart rates during supraventricular tachycardia in our patients were greater than 270 beats/minute. It may be difficult, however, to differentiate clinically between a tachycardia of 200-240/minute (which may be sinus tachycardia) and a rate of 250 and above, which is always an arrhythmia. For this reason, when there is any doubt, an electrocardiogram should be carried out so that the heart rate can be accurately measured. Some electrocardiographic monitors are unable to count accurately at rates above 220 or 230 / minute and should not be relied upon for an accurate measurement of the heart rate.

Two of our patients (both of whom were pre- 
mature) had heart rates of 215 and $250 /$ minute, respectively, during tachycardia. This accords with previous reports of lower tachycardia rates in the fetus and in prematurity, although the reason for the difference in heart rates in supraventricular tachycardia between premature babies and those born at full term is unclear. ${ }^{9}$

The ideal treatment for supraventricular tachycardia in early infancy (or for that matter at any age) would be safe, rapidly effective in restoring sinus rhythm, easily carried out or given, free of side effects, and would prevent a recurrence of the tachycardia or be easily repeated if tachycardia recurred. Until fairly recently most patients were treated with intravenous or intramuscular digoxin or with cardioversion. Both of these treatments failed to approach the ideal, but stimulated interest in newer drugs or other forms of treatment.

Digoxin was effective in only half our patients and there was a mean delay of 13 hours before restoration of sinus rhythm. Previous studies have shown that digoxin is effective in up to $87 \%$ of patients. ${ }^{10}$ In the present study $20 \%$ of patients given digoxin in the referring hospital received appreciable overdoses. Miscalculation of paediatric doses of digoxin is a common error, easily made, ${ }^{11}$ and there is a wide variation in the recommended doses for infants.

Cardioversion when carried out correctly is safe and effective. Recurrence of tachycardia is common, however, and as the procedure is performed under brief general anaesthesia it is not easy to repeat. The dose should be restricted to $0.5-1 \mathrm{~J} / \mathrm{kg}^{6}$

The dangers of giving verapamil to infants are now well recognised. ${ }^{5}$ Although many patients may be safely and effectively treated with verapamil, the adverse effects are unpredictable and the drug is best avoided in this age group. ${ }^{6}$

Because treatment with iced water has proved to be so effective we have little experience with intravenous adenosine in this age group. Other workers have reported high success rates, however, low incidences of side effects, and rapid action, ${ }^{12}$ but there is an appreciable incidence of recurrence of tachycardia.

In our experience the facial application of iced water, either with an ice pack or by immersion of the face comes close to the ideal form of treatment. It was rapidly effective on $90 \%$ of the occasions on which it was attempted, was easily carried out, and was safe with no side effects. Recurrence of tachycardia was fairly common, but the treatment is easy to repeat. The success rate of the procedure was higher in this hospital than in referring hospitals, and with wider experience among general paediatricians it should become the treatment of choice for supraventricular tachycardia in neonates or in early infancy.

We thank Dr HH Bain and Dr S Hunter for permission to report details of patients under their care.

1 Roberton NRC. Textbook of neonatalogy. Edinburgh: Churchill Livingstone, 1986:379.

2 Milner AD, Hull D. Hospital paediatrics. Edinburgh: Churchill Livingstone, 1984:120-1.

3 Roberton NRC. Manual of neonatal intensive care. London: Edward Arnold, 1986:163-4.

4 Wilkinson JL. Management of paroxysmal tachycardia. Arch Dis Child 1983;58:945-6.

5 Radford D. Side effects of verapamil in infants. Arch Dis Child 1983;58:465-6.

6 Garson A Jr. Medicolegal problems in the management of cardiac arrhythmias in children. Pediatrics 1987;79:84-8.

Sperandeo V, Pieri D, Palazzolo P, Donzelli M, Spataro G. Sundeo V, Pieri D, Palazzolo P, Donzelli M, Spataro G. Supraventricular tachycardia in infants:

8 Whitman V, Friedman L, Berman W Jr, Maisels MJ. Supraventricular tachycardia in newborn infants. An approach to ventricular tachycardia in newborn

9 Zales VR, Dunnigan A, Benson DW Jr. Clinical and electrocardiographic features of fetal and neonatal paroxysmal atrial tachycardia resulting in congestive heart failure. Am 7 Cardiol 1988;62:225-8.

10 Deal BJ, Keane JF, Gillette PC, Garson A Jr. WolffParkinson-White syndrome and supraventricular tachycardia during infancy: management and follow up. $\mathcal{F} \mathrm{Am} \mathrm{Coll}$ Cardiol 1985;5:130-5.

11 Annual Report of the Council. Paediatric overdose of digoxin London: The Medical Protection Society, 1988:28.

12 Till J, Shinebourne EA, Rigby M, Clarke B, Ward DE, Rowland $E$. Adenosine: efficacy and safety in the treatment of supraventricular tachycardia in infants and children. $\mathrm{Br}$ Heart $\mathcal{f}$ 1989;62:204-11. 